\title{
Sex differences in gut microbiota in patients with major depressive disorder
}

This article was published in the following Dove Press journal:

Neuropsychiatric Disease and Treatment

\author{
Jian-jun Chen ${ }^{1-4}$ \\ Peng Zheng ${ }^{2,3}$ \\ Yi-yun Liu ${ }^{2,3}$ \\ Xiao-gang Zhong ${ }^{2,3}$ \\ Hai-yang Wang ${ }^{2,3}$ \\ Yu-jie Guo 2,3 \\ Peng $\mathrm{Xie}^{2,3}$ \\ 'Institute of Life Sciences, \\ ${ }^{2}$ Department of Neurology, First \\ Affiliated Hospital, ${ }^{3}$ nnstitute of \\ Neuroscience, ${ }^{4}$ Joint International \\ Research Laboratory of Reproduction \\ and Development, Chongqing Medical \\ University, Chongqing, China
}

Correspondence: Peng Xie

Department of Neurology, First Affiliated Hospital, Chongqing Medical University, I Yixueyuan Road, Yuzhong, Chongqing 4000 I6, China

Tel +862368485490

Fax +86236848 5I II

Email xiepeng@cqmu.edu.cn
Objective: Our previous studies found that disturbances in gut microbiota might have a causative role in the onset of major depressive disorder (MDD). The aim of this study was to investigate whether there were sex differences in gut microbiota in patients with MDD.

Patients and methods: First-episode drug-naïve MDD patients and healthy controls were included. 16S rRNA gene sequences extracted from the fecal samples of the included subjects were analyzed. Principal-coordinate analysis and partial least squares-discriminant analysis were used to assess whether there were sex-specific gut microbiota. A random forest algorithm was used to identify the differential operational taxonomic units. Linear discriminant-analysis effect size was further used to identify the dominant sex-specific phylotypes responsible for the differences between MDD patients and healthy controls.

Results: In total, 57 and 74 differential operational taxonomic units responsible for separating female and male MDD patients from their healthy counterparts were identified. Compared with their healthy counterparts, increased Actinobacteria and decreased Bacteroidetes levels were found in female and male MDD patients, respectively. The most differentially abundant bacterial taxa in female and male MDD patients belonged to phyla Actinobacteria and Bacteroidia, respectively. Meanwhile, female and male MDD patients had different dominant phylotypes. Conclusion: These results demonstrated that there were sex differences in gut microbiota in patients with MDD. The suitability of Actinobacteria and Bacteroidia as the sex-specific biomarkers for diagnosing MDD should be further explored.

Keywords: major depressive disorder, MDD, gut microbiota, biomarker

\section{Introduction}

Major depressive disorder (MDD) is a mental disorder characterized by loss of interest in normally enjoyable activities, low self-esteem, and low energy. This disease imposes a huge economic burden on the whole society. The pathogenesis of MDD is still unclear, and there are no objective diagnostic methods or $100 \%$-effective treatment methods. ${ }^{1,2}$ Many factors, such as genetics, biochemical or neurophysiological changes, and psychosocial variables, are associated with MDD. ${ }^{3,4}$ Recently, many researchers have attempted to explain its pathogenesis using neuroanatomical abnormalities, neurotransmission deficiency, and neurotrophic alterations. ${ }^{5}$ However, none of these theories has been universally accepted. Therefore, there is an urgent need to identify novel pathophysiologic mechanisms underlying MDD.

A previous study reported that gut microbiota could influence all aspects of physiology. ${ }^{6}$ Many diseases have been found to be related to disturbed gut microbiota, such as obesity and diabetes. ${ }^{7,8}$ Researchers have also found that gut microbiota had an influence on brain function and behavior through the microbiota-gut-brain axis., ${ }^{9,10}$ Our previous study proved that gut microbiota could influence the expression levels of 
genes in the hippocampi of mice. ${ }^{11}$ Meanwhile, our previous metabolomic studies showed an interesting phenomenon wherein several differential metabolites in MDD patients were the metabolic byproducts of gut microbiota. ${ }^{12,13}$ Moreover, clinical studies have reported disturbed gut microbiota in limited samples of depressed patients. ${ }^{14,15}$ Based on these results, we conducted a further study, and found that the dysbiosis of gut microbiota might be a contributory factor in the development of MDD. ${ }^{16}$

Nowadays, the disproportionate prevalence of MDD in women might be the most consistent finding among studies on MDD. In our previous study, compared to healthy controls (HCs), the relative abundance of Bacteroidetes and Actinobacteria were found to be significantly changed in patients with MDD. ${ }^{16}$ However, possible sex differences in gut microbiota were not taken into consideration. Actually, our previous metabolomic study found that there were divergent urinary metabolic phenotypes between males and females with MDD. ${ }^{17}$ Yurkovetskiy et al reported that hormonal regulation of microbe-controlling mechanisms could result in differences in microbial composition between males and females. ${ }^{18}$ Moreover, a recently published study reported that there were sex-specific transcriptional signatures in human depression. ${ }^{19}$ Therefore, we hypothesized that there were sex differences in gut microbiota in patients with MDD, and conducted this study to identify sex-specific gut microbiota.

\section{Patients and methods Subject recruitment}

The protocol of this study was reviewed and approved by the ethical committee of Chongqing Medical University (Chongqing, China). The methods were carried out in accordance with approved guidelines and regulations. The 17-item Hamilton Depression Rating Scale (HDRS-17) was used to assess depression severity. ${ }^{20}$ Two experienced psychiatrists studying and treating depression for several years systematically used the HDRS-17 to diagnose each participant. MDD patients who met the following criteria were included: $\geq 18$ years old, first-episode drug-naïve, and without obesity, diabetes, substance abuse, preexisting physical diseases, or other mental disorders. HCs did not have any previous lifetime history of Diagnostic and Statistical Manual of Mental Disorders IV axis I/II neurological diseases or systemic medical illness. MDD patients receiving nonpharmacologic treatments were also excluded. Subjects using antibiotics or probiotics were excluded. Pregnant, nursing, or currently menstruating candidates were excluded. All subjects recruited provided written informed consent.
MDD patients and HCs were recruited from the psychiatric center and medical examination center, respectively.

\section{I6S rRNA gene sequencing}

Fecal samples were collected and stored at $-80^{\circ} \mathrm{C}$ prior to analysis. The standard PowerSoil kit protocol was used to extract bacterial genomic DNA. Briefly, the frozen samples were thawed on ice and then pulverized with a pestle and mortar in liquid nitrogen, we added MoBio lysis buffer to these fecal samples and then mixed them, and after centrifuging, we placed the obtained supernatant into MoBio garnet-bead tubes containing MoBio buffer. Extracted V3-V5 regions of the 16S rRNA gene from these fecal samples were amplified by polymerase chain reaction with bar-coded universal primers containing linker sequences for pyrosequencing. ${ }^{21}$ The 454 sequencing system (Hoffman-La Roche, Basel, Switzerland) was used.

\section{I6S rRNA gene-sequencing analysis}

In order to obtain unique reads, Mothur 1.31.2 was used to quality-filter the raw sequences obtained from 454 sequencing. ${ }^{22}$ Sequences meeting any one of the following criteria were excluded: $<200 \mathrm{bp}$ or $>1,000 \mathrm{bp}$, contained any bar-code mismatches, primer mismatches, or ambiguous bases, and contained homopolymer runs exceeding six bases. Finally, the remaining sequences were assigned to operational taxonomic units (OTUs) and then taxonomically classified using RDP reference database. ${ }^{23}$ To calculate the relative abundance of gut microbiota at different levels, summaries of taxonomic distributions of OTUs were constructed using these taxonomies. Four parameters (observed species, phylogenetic diversity, Shannon index, Simpson index) were used to calculate $\alpha$-diversity. ${ }^{24} \beta$-Diversity was reported according to principal-coordinate analysis (PCoA). ${ }^{25}$ Meanwhile, both PCoA and partial least squares-discriminant analysis (PLS-DA) were used to find out whether MDD patients could be separated from HCs. A random forest algorithm was used to identify the differential OTUs responsible for sample differentiation. Cytoscape 3.2.1 software was used to analyze the potential relationship between demographic data and differential OTUs. Finally, the linear discriminantanalysis effect size (LEfSe) was further used to identify the dominant sex-specific phylotypes responsible for differences between MDD patients and $\mathrm{HCs} .{ }^{26}$

\section{Results}

\section{Demographic data}

A total of 24 first-episode drug-naïve female MDD patients and 24 demographically matched female HCs were recruited. 
The average ages of these MDD patients and HCs were $41.5 \pm 11.53$ and $43.95 \pm 12.11$ years $(P=0.475)$, respectively. These MDD patients $\left(22.01 \pm 2.17 \mathrm{~kg} / \mathrm{m}^{2}\right)$ and HCs $\left(22.63 \pm 2.43 \mathrm{~kg} / \mathrm{m}^{2}\right)$ had similar average body-mass index (BMI; $P=0.356$ ). The average HDRS scores of these MDD patients were 23.04 \pm 4.93 . Meanwhile, 20 first-episode drug-naïve male MDD patients and 20 demographically matched male HCs were recruited. The average ages of these MDD patients and HCs were 40.35 \pm 11.05 and $42.80 \pm 15.13$ years $(P=0.562)$, respectively. These MDD patients $\left(22.22 \pm 2.18 \mathrm{~kg} / \mathrm{m}^{2}\right)$ and HCs $\left(22.50 \pm 2.25 \mathrm{~kg} / \mathrm{m}^{2}\right)$ had similar average BMI $(P=0.694)$. The average HDRS scores of these MDD patients were 23.9 \pm 3.68 . Three female and two male MDD patients had coexisting anxiety disorders.

\section{Disturbed gut microbiota}

The majority of the obtained OTUs belonged to four phyla (female vs male): Firmicutes ( $73 \%$ vs $75.2 \%$ ), Bacteroidetes ( $10 \%$ vs $9.7 \%)$, Actinobacteria (6.5\% vs $6.8 \%$ ), and Proteobacteria $(4.8 \%$ vs $5.3 \%)$. The results of within-sample $(\alpha)$ phylogenetic diversity analysis showed no significant differences between MDD patients and HCs. The results of PCoA showed that gut microbial community composition was significantly different between female MDD patients and HCs (Figure 1A). The PLS-DA model showed similar results (Figure 1B). Meanwhile, the PCoA and PLS-DA models also showed significant differences in gut microbial community composition between male MDD patients and HCs (Figure 2).

A

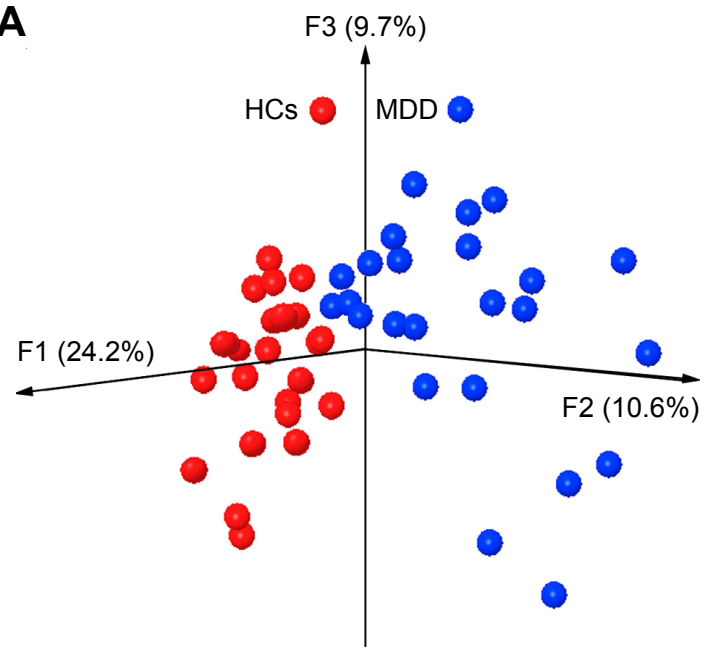

\section{Differential gut microbiota}

A random forest classifier was used to identify the key discriminatory OTUs responsible for separating MDD patients from HCs. In total, 57 OTUs whose relative abundance could reliably distinguish female MDD patients from female HCs were identified. Of these differential OTUs, the 29 increased OTUs in female MDD patients were mainly assigned to the families of Coriobacteriaceae, Lachnospiraceae, and Ruminococcaceae, and the 28 decreased OTUs were mainly assigned to the families of Lachnospiraceae and Ruminococcaceae (Figure 3). These differential OTUs were mainly assigned to the phyla Firmicutes (45 of 57, 78.9\%), Actinobacteria (six of 57, 10.5\%), and Bacteroidetes (three of 57, 5.3\%). Meanwhile, 74 OTUs whose relative abundance could reliably distinguish male MDD patients from HCs were identified. Of these differential OTUs, the 21 increased OTUs in male MDD patients were mainly assigned to the families of Lachnospiraceae and Erysipelotrichaceae, and the decreased 53 OTUs were mainly assigned to the families of Lachnospiraceae and Ruminococcaceae (Figure 4). The phyla these were mainly assigned to were Firmicutes (62 of 74, $83.8 \%$ ), Actinobacteria (three of $74,4 \%$ ), and Bacteroidetes (six of 74, 8.1\%). Compared with female HCs, the relative abundance of Actinobacteria was increased in female MDD patients. Compared with male HCs, the relative abundance of Bacteroidetes was decreased in male MDD patients.

\section{Dominant phylotypes}

Dominant phylotypes responsible for the differences between MDD patients and HCs were identified by the metagenomic
B

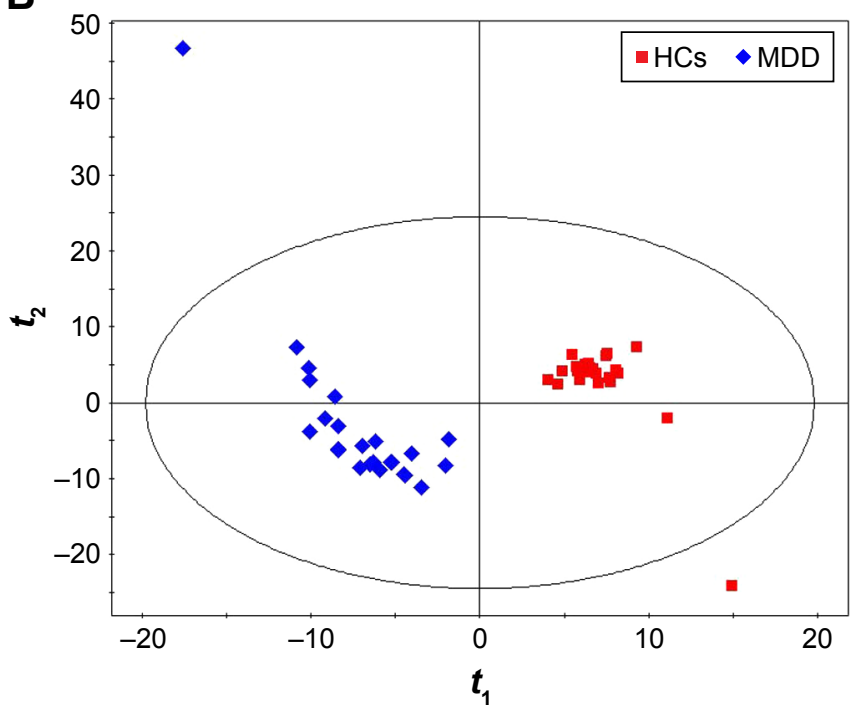

Figure I Obvious differences in gut microbial composition between female MDD patients and HCs.

Notes: (A) Three-dimensional principal-coordinate analysis; (B) partial least squares-discriminant analysis.

Abbreviations: MDD, major depressive disorder; $\mathrm{HCs}$, healthy controls. 
A

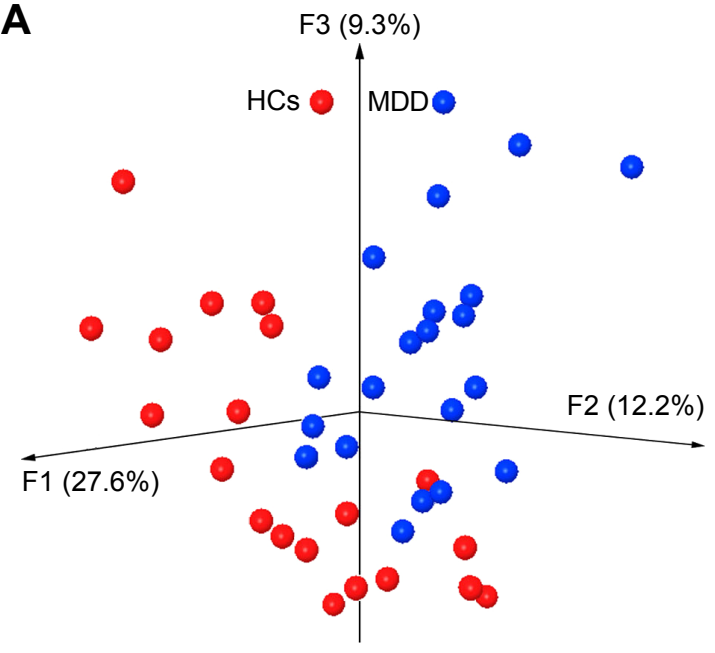

B

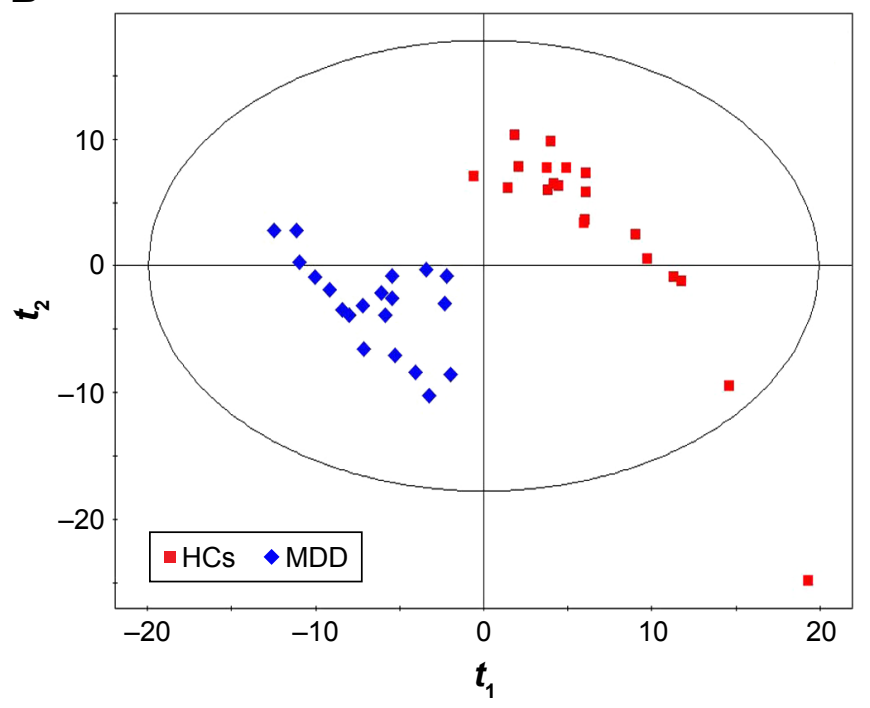

Figure 2 Obvious differences in gut microbial composition between male MDD patients and HCs.

Notes: (A) Three-dimensional principal-coordinate analysis; (B) partial least squares-discriminant analysis.

Abbreviations: MDD, major depressive disorder; $\mathrm{HCs}$, healthy controls.

LEfSe approach. LEfSe is a new method for metagenomic biomarker discovery by way of class comparison. In total, 22 bacterial clades with statistically significant and biologically consistent differences in female MDD patients were identified (Figure 5A). The most differentially abundant bacterial taxa in female MDD patients belonged to Actinobacteria. At the genus level, Actinomyces, Bifidobacterium, Asaccharobacter, Atopobium, Eggerthella, Gordonibacter, Olsenella, Eubacterium, Anaerostipes, Blautia, Roseburia, Faecalibacterium, and Desulfovibrio, which were most abundant in female MDD patients, and Howardella, Sutterella, and
Pyramidobacter, which were most abundant in female HCs, were the key phylotypes that contributed to the different gut microbiota between female MDD patients and HCs (Figure 5B).

Meanwhile, six bacterial clades with statistically significant and biologically consistent differences in male MDD patients were identified (Figure 6A). The most differentially abundant bacterial taxa in male MDD patients belonged to Bacteroidia (Bacteroidetes). At the genus level, Bacteroides, Erysipelotrichaceae incertae sedis, Veillonella, and Atopobium, which were most abundant in male MDD patients, and

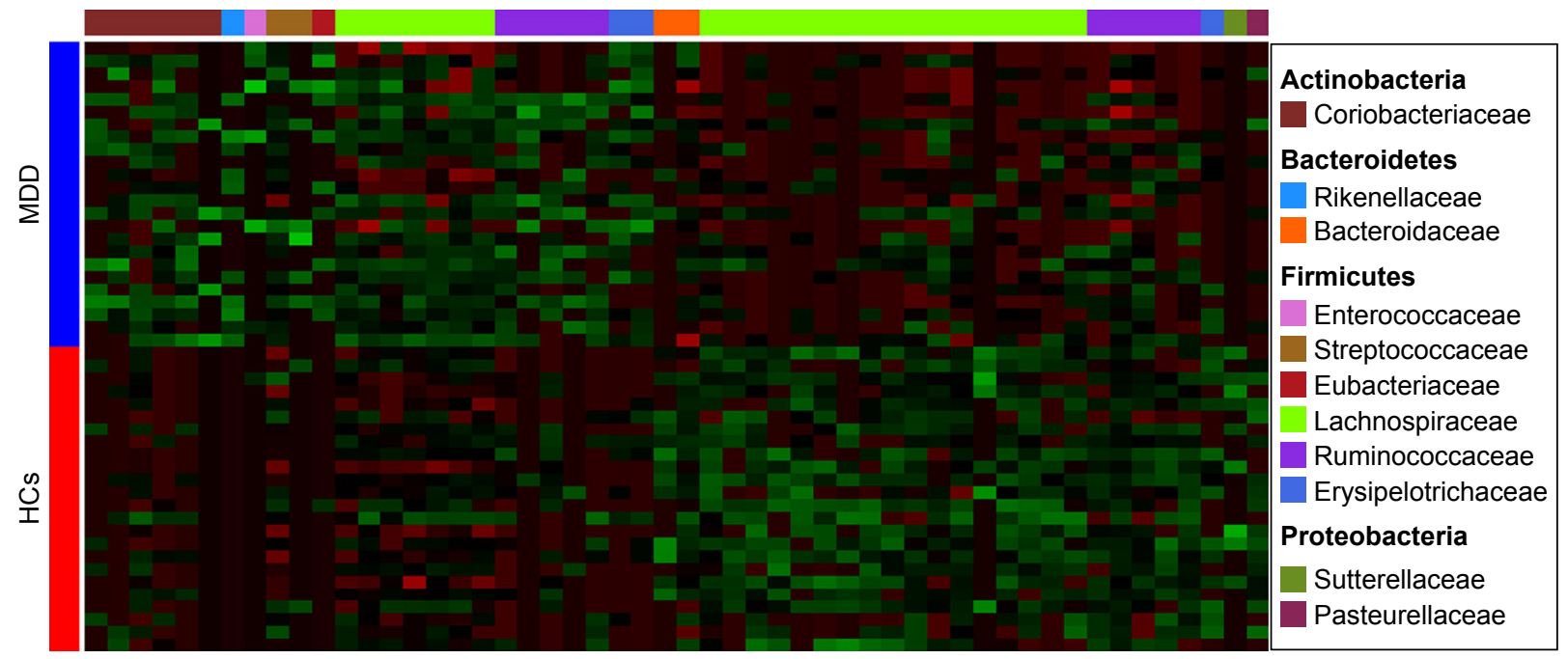

Figure 3 Heat map of differential operational taxonomic unit abundance between female MDD patients and HCs.

Notes: Assignment of each operational taxonomic unit provided at right. Green and red indicate increase and decrease, respectively.

Abbreviations: MDD, major depressive disorder; HCs, healthy controls. 


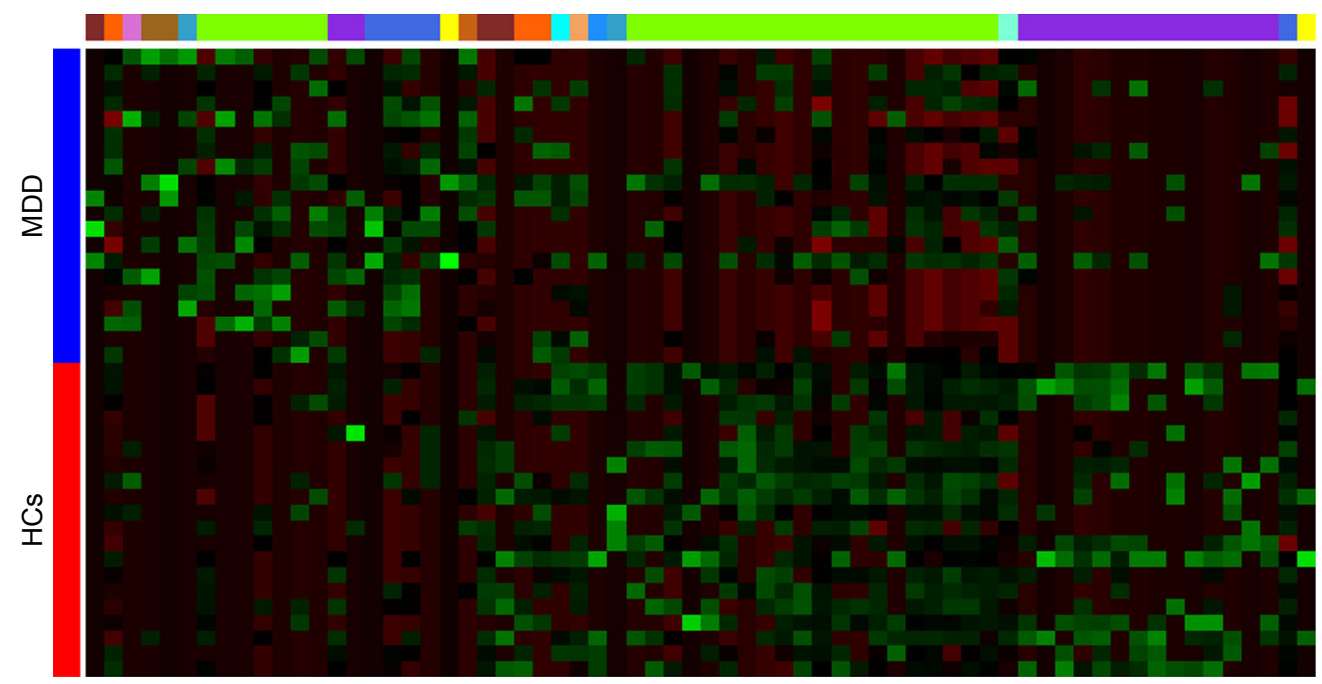

\begin{tabular}{|l|}
\hline Actinobacteria \\
Coriobacteriaceae \\
Bacteroidetes \\
Rikenellaceae \\
Bacteroidaceae \\
Porphyromonadaceae \\
Prevotellaceae \\
Firmicutes \\
Enterococcaceae \\
Streptococcaceae \\
Lachnospiraceae \\
Ruminococcaceae \\
Erysipelotrichaceae \\
Clostridiaceae 1 \\
Peptostreptococcaceae \\
Acidaminococcaceae \\
Veillonellaceae \\
\hline
\end{tabular}

Figure 4 Heat map of differential operational taxonomic unit abundance between male MDD patients and HCs.

Notes: Assignment of each operational taxonomic unit provided at right. Green and red indicate increase and decrease, respectively. Abbreviations: MDD, major depressive disorder; $\mathrm{HCs}$, healthy controls.

Anaerovorax, Gordonibacter, and Pyramidobacter, which were most abundant in male HCs, were the key phylotypes that contributed to the different gut microbiota between male MDD patients and HCs (Figure 6B).

\section{Correlation analysis}

CoNet 1.1 (Cytoscape application) was used to evaluate correlations among the demographic data and relative abundance of bacterial genera (Figure 7). For female MDD patients, six genera (Asaccharobacter, Clostridium XIVa,
Erysipelotrichaceae incertae sedis, Streptococcus, Faecalibacterium, and Lachnospira incertae sedis) were negatively correlated with age, three genera (Clostridium XIVa, Erysipelotrichaceae incertae sedis, and Streptococcus) were negatively correlated with HDRS score, and one genus (Streptococcus) was negatively correlated with BMI. For male MDD patients, one genus (Erysipelotrichaceae incertae sedis) was negatively correlated with age, two genera (Veillonella and Collinsella) were negatively and positively, respectively, correlated with HDRS score, and

\section{A}

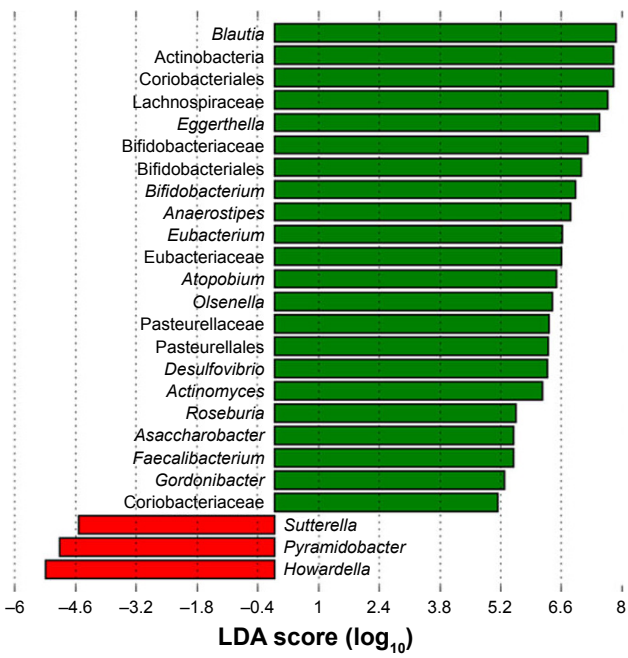

B

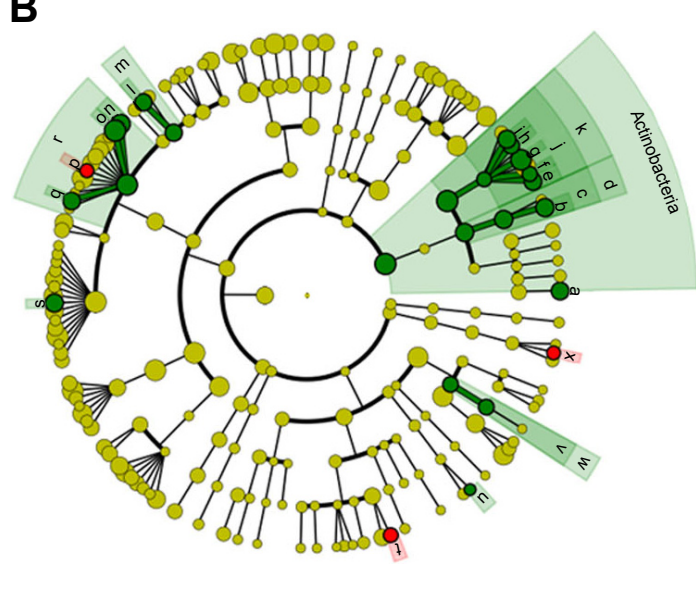

a: Actinomyces b: Bifidobacterium c: Bifidobacteriacea e: Asaccharobacter $\square \mathrm{f:}$ Atopobium g: Eggerthella h: Gordonibacter $\square$ i: Olsenella j: Coriobacteriacea k: Coriobacterial I: Eubacterium m:Eubacteriaceae $\square$ o: Blautia p: Howardella q: Roseburia r: Lachnospiracea s: Faecalibacterium t: Sutterella u: Desulfovibrio v: Pasteurellaceae w: Pasteurellales x: Pyramidobacter

Figure $\mathbf{5}$ Taxonomic differences in gut microbiota in female subjects.

Notes: (A) Bacterial clades (25) with statistically significant and biologically consistent differences in female MDD patients and HCs (LDA score >2); (B) MDD-enriched taxa indicated by positive LDA scores (green), and HC-enriched taxa indicated by negative scores (red).

Abbreviations: MDD, major depressive disorder; HCs, healthy controls; LDA, linear discriminant analysis. 
A

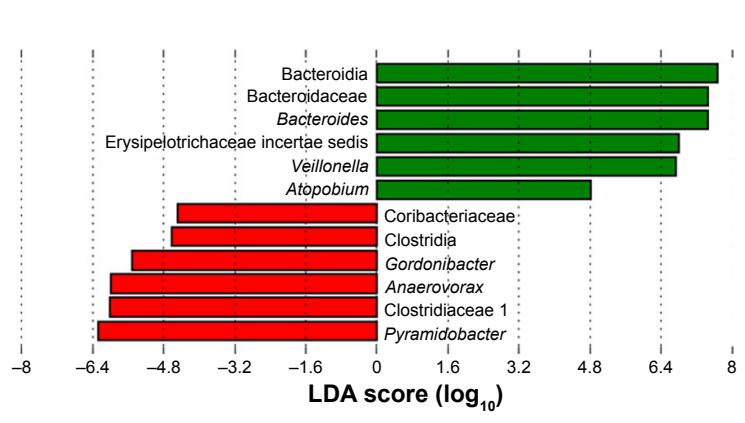

B

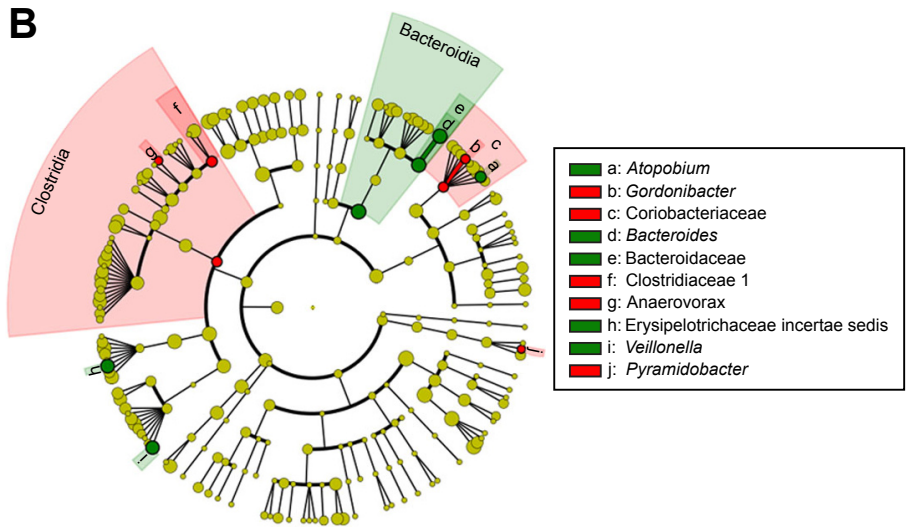

$\varpi$ HCs $\square$ MDD

Figure 6 Taxonomic differences in gut microbiota in male subjects.

Notes: (A) Twelve bacterial clades with statistically significant and biologically consistent differences in male MDD patients and HCs (LDA score >2); (B) MDD-enriched taxa indicated by positive LDA scores (green), and HC-enriched taxa indicated by negative scores (red).

Abbreviations: MDD, major depressive disorder; HCs, healthy controls; LDA, linear discriminant analysis.

two genera (Dorea and Lachnospira incertae sedis) were positively correlated with BMI. These results showed that only three key phylotypes (Erysipelotrichaceae incertae sedis, Asaccharobacter, and Faecalibacterium) were negatively correlated with age and one key phylotype (Veillonella) was negatively correlated with HDRS, while other key phylotypes showed no correlation with demographic data.

\section{Discussion}

In total, 57 female-specific and 74 male-specific differential OTUs were identified. Among these OTUs, only 18 differential
OTUs (mainly assigned to the phyla Firmicutes, 14 of 18 , $77.8 \%$ ) were present in both female and male MDD patients. The relative abundance of Actinobacteria and Bacteroidetes was increased and decreased in female and male MDD patients compared to their healthy counterparts, respectively. Moreover, the most differentially abundant bacterial taxa belonged to Actinobacteria in female MDD patients and Bacteroidia (phyla Bacteroidetes) in male MDD patients. The dominant phylotypes in female and male MDD patients were also different. These results demonstrated that there were sex differences in gut microbiota in patients with MDD,
A

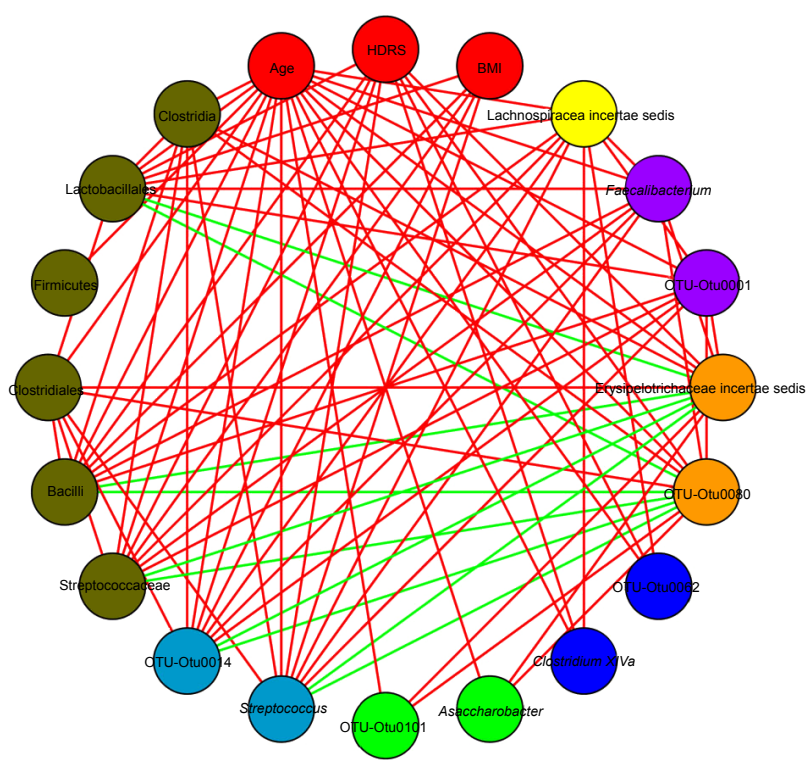

B

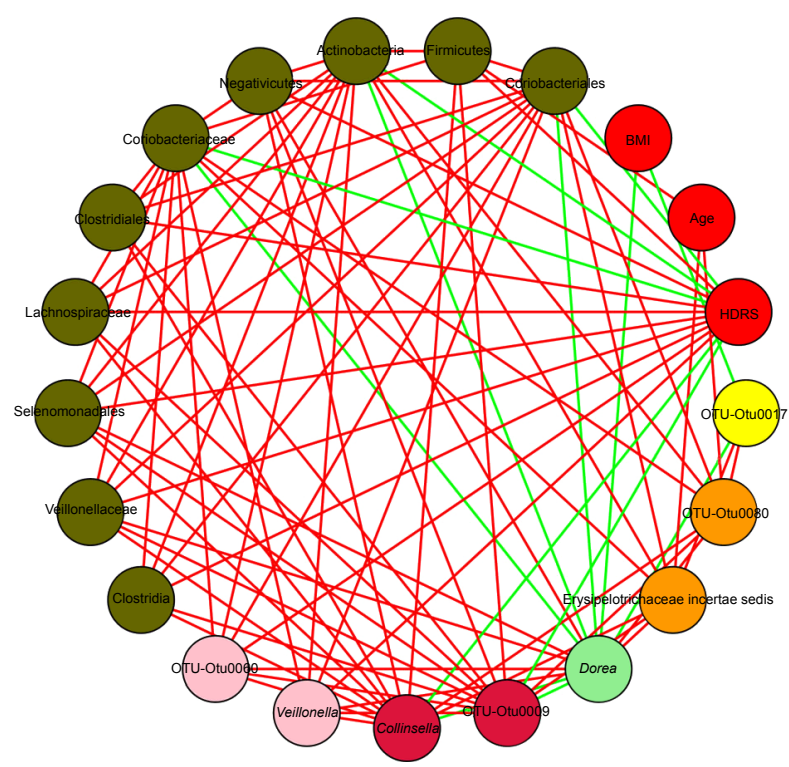

Figure 7 Associations among demographic data (age, BMI, and HDRS) and gut microbiota.

Notes: (A) Female and (B) male MDD patients. Red lines indicate positive relationships, green lines negative relationship, and circles: red, demographic data; green, Asaccharobacter; blue, Clostridium XIVa; orange, Erysipelotrichaceae incertae sedis; dark orchid, Faecalibacterium; yellow, Lachnospiraceae incertae sedis; deep sky blue, Streptococcus; crimson, Collinsella; light green, Dorea; pink, Veillonella; olive, others.

Abbreviations: BMI, body-mass index; HDRS, Hamilton Depression Rating Scale; MDD, major depressive disorder. 
and the suitability of Actinobacteria and Bacteroidia as the sex-specific biomarkers for diagnosing MDD should be further evaluated.

Our previous study found that the relative abundance of Bacteroidetes and Actinobacteria was significantly decreased and increased, respectively, in MDD patients. ${ }^{16}$ The purpose of this previous study was to investigate whether the dysbiosis of the gut microbiome played a causal role in the development of depressive-like behaviors. Therefore, sex-based differences were not taken into consideration. However, sexbased differences are prominent in affective disorders. ${ }^{27-29}$ Our previous metabolomic studies also found sex-specific differential metabolites in MDD and bipolar disorder. ${ }^{17,30}$ Therefore, when separately analyzing the differential gut microbiota in female and male MDD patients, we found that the Bacteroidetes level was only significantly decreased in male MDD patients and the Actinobacteria level only significantly increased in female MDD patients. Moreover, in order to rule out the potential influence of antidepressants on gut microbiota, only drug-naïve MDD patients were recruited, which might make our conclusion more robust in identifying sex-specific gut microbiota.

A previous study reported a general underrepresentation of Bacteroidetes related with depression. ${ }^{14}$ However, Jiang et al found that Bacteroidetes levels were increased in MDD patients. ${ }^{15}$ In this study, decreased Bacteroidetes levels were found in male MDD patients. Meanwhile, Jiang et al also reported the decreased phyla Firmicutes level and increased phyla Actinobacteria level in MDD patients. However our results showed that increased Actinobacteria levels were only found in female MDD patients, and the overall relative abundance of Firmicutes was not significantly changed in female or male MDD patients. This disparity might be caused by the sex factor. Additionally, the differences in demographic and clinical characteristics of the recruited MDD patients, sample sizes, and/or statistical methods used to identify MDD-associated gut microbiota might also have a role in this disparity. However, these results consistently showed that MDD was linked to distinct alterations in gut microbial compositions.

In clinical practice, depression and metabolic-disease comorbidity, such as diabetes and obesity, is common. ${ }^{31}$ Previous studies have shown that low Bacteroidetes levels were associated with obesity. ${ }^{32,33}$ Stunkard et al reported a link between depression and obesity through low-grade inflammation. ${ }^{34}$ Meanwhile, Troseid et al established a correlation between low-grade inflammation and bacteria. ${ }^{35}$ Therefore, in order to rule out the potential influence of obesity and diabetes, we excluded subjects with either. Finally, the difference in BMI between MDD patients and HCs was almost negligible. Moreover, the correlation analysis also showed that there was no correlation between any key phylotypes and BMI. It is unlikely that obesity or diabetes could be a confounding factor in this study.

Although no significant differences in the overall abundance of Firmicutes between HCs and MDD patients were identified in this study, some Firmicutes OTUs in both female and male MDD patients were increased, while others were decreased. Therefore, the disturbed Firmicutes could still be a hallmark in MDD. ${ }^{16}$ Moreover, the correlation analysis found that the relative abundance of seven genera (Clostridium XIVa, Erysipelotrichaceae incertae sedis, Streptococcus, Dorea, Faecalibacterium, Lachnospira incertae sedis, and Veillonella) belonging to Firmicutes showed correlations with age, HDRS score, and BMI. A previous study reported that there was a negative relationship between the severity of depressive symptoms and the relative abundance of Faecalibacterium, ${ }^{15}$ but in this study only the negative relationship between the severity of depressive symptoms and the relative abundance of four other genera (Clostridium XIVa, Streptococcus, Erysipelotrichaceae incertae sedis, and Veillonella) was identified. These different results might also be caused by the sex factor. In addition, a positive relationship between the severity of depressive symptoms and the relative abundance of Collinsella in male MDD patients was found here. Schnorr and Bachner reported a reduction in Actinobacteria levels after intervention, mainly from the loss of Collinsella. ${ }^{36}$ As such, Collinsella might also be a useful index for the clinical management and treatment of MDD.

The brain can alter gut function, and is widely acknowledged. However, it is less readily accepted that signals from the gut can influence brain function. Actually, gut microbiota could regulate the size and composition of bile-acid pool size, and in turn be an important regulator of the bloodbrain barrier and hypothalamic-pituitary-adrenal axis. ${ }^{37}$ Meanwhile, metabolites from the gut microbiota have a significant effect on regulating the gut-brain axis and host immunity. ${ }^{38}$ Gut microbiota can also regulate brain function by influencing tryptophan metabolism ${ }^{39}$ and influence the development and activity of brain tissue by regulating microglia homoeostasis. ${ }^{40}$ In addition, Sobko et al found that Lactobacillus could convert nitrate to nitric oxide, which is a potent regulator of the immune and nervous systems. ${ }^{41}$ Previous studies have reported that L. rhamnosus and L. acidophilus might have an analgesic action on the host by inhibiting the spinal neuron cellular memory of the distension. ${ }^{42,43}$ 


\section{Limitations}

Limitations should be noticed here. First, the number of recruited samples was relatively small, and the results of this exploratory research need future studies to verify and support them. Second, an animal experiment was not performed to determine whether Actinobacteria or Bacteroidetes could be potential targets for MDD treatment. Third, a previous study reported that dietary habits could influence gut microbiota, ${ }^{44}$ but the relationship between dietary intake and gut microbial compositions could not be analyzed here, because of missing detailed dietary information. However, our findings were not likely to be significantly influenced by this potential confounding factor, because of the similar lifestyles and dietary habits of the recruited samples from Chongqing. Fourth, all recruited subjects were from the same place, and thus ethnic biases and site-specificity in microbial phenotypes could not be ruled out. Fifth, we did not measure estrogen levels in female patients, but Baker et al reported that gut microbiota could regulate estrogen levels through secretion of $\beta$-glucuronidase. ${ }^{45}$ Future studies should explore the potential relationship between estrogen levels and gut microbiota. Finally, we did not analyze depressive episode duration, although previous a study found that there was evidence for the interplay between immune and endocrine systems in drug-naïve MDD patients with short-illnessduration first affective episodes. ${ }^{46}$

\section{Conclusion}

This study firstly determined that there were sex differences in gut microbiota in patients with MDD and identified genderspecific gut microbiota. The most differentially abundant bacterial taxa in female and male MDD patients belonged to Actinobacteria and Bacteroidia, respectively. These findings could provide new insights for uncovering the pathogenesis of MDD and studying potential gut-mediated therapies for MDD. However, due to the risk of overseeing effects in small cohorts, these findings must be verified and supported in the larger cohorts. Meanwhile, future studies are warranted to evaluate the suitability of Actinobacteria and Bacteroidia as sex-specific biomarkers for diagnosing MDD.

\section{Acknowledgments}

This work was supported by the Natural Science Foundation Project of China (81701360, 81601208, 81771490), Chongqing Science and Technology Commission (cstc2017jcyjA0207, cstc2014kjrc-qnrc10004), Science and Technology Research Program of Chongqing Municipal Education Commission (grant KJ1702037), Special Project on Natural
Chronic Noninfectious Diseases (2016YFC1307200), National Key Research and Development Program of China (2017YFA0505700), and National Basic Research Program of China (973 Program, grant 2009CB918300).

\section{Disclosure}

The authors report no conflicts of interest in this work.

\section{References}

1. Chen JJ, Zhou CJ, Zheng P, et al. Differential urinary metabolites related with the severity of major depressive disorder. Behav Brain Res. 2017;332:280-287.

2. Chen JJ, Zhao LB, Liu YY, Fan SH, Xie P. Comparative efficacy and acceptability of electroconvulsive therapy versus repetitive transcranial magnetic stimulation for major depression: a systematic review and multiple-treatments meta-analysis. Behav Brain Res. 2017;320:30-36.

3. Belmaker RH, Agam G. Major depressive disorder. $N$ Engl J Med. 2008;358:55-68.

4. Chen JJ, Zhou CJ, Liu Z, et al. Divergent urinary metabolic phenotypes between major depressive disorder and bipolar disorder identified by a combined GC-MS and NMR spectroscopic metabonomic approach. J Proteome Res. 2015;14(8):3382-3389.

5. Guilloux JP, Douillard-Guilloux G, Kota R, et al. Molecular evidence for BDNF- and GABA-related dysfunctions in the amygdala of female subjects with major depression. Mol Psychiatry. 2012;17:1130-1142.

6. Clemente JC, Ursell LK, Parfrey LW, Knight R. The impact of the gut microbiota on human health: an integrative view. Cell. 2012;148: $1258-1270$

7. Wen L, Ley RE, Volchkov PY, et al. Innate immunity and intestinal microbiota in the development of type 1 diabetes. Nature. 2008;455: $1109-1113$.

8. Ridaura VK, Faith JJ, Rey FE, et al. Gut microbiota from twins discordant for obesity modulate metabolism in mice. Science. 2013;341: 1241214.

9. Bercik P, Denou E, Collins J, et al. The intestinal microbiota affect central levels of brain-derived neurotropic factor and behavior in mice. Gastroenterology. 2011;141:599-609.

10. Clarke G, Grenham S, Scully P, et al. The microbiome-gut-brain axis during early life regulates the hippocampal serotonergic system in a sex-dependent manner. Mol Psychiatry. 2013;18:666-673.

11. Chen J, Zeng B, Li W, et al. Effects of gut microbiota on the microRNA and mRNA expression in the hippocampus of mice. Behav Brain Res. 2017;322:34-41.

12. Zheng P, Wang Y, Chen L, et al. Identification and validation of urinary metabolite biomarkers for major depressive disorder. Mol Cell Proteomics. 2013;12:207-214.

13. Zheng P, Chen JJ, Huang T, et al. A novel urinary metabolite signature for diagnosing major depressive disorder. J Proteome Res. 2013;12: 5904-5911.

14. Naseribafrouei A, Hestad K, Avershina E, et al. Correlation between the human fecal microbiota and depression. Neurogastroenterol Motil. 2014;26:1155-1162.

15. Jiang $\mathrm{H}$, Ling Z, Zhang $\mathrm{Y}$, et al. Altered fecal microbiota composition in patients with major depressive disorder. Brain Behav Immun. 2015; 48:186-194

16. Zheng P, Zeng B, Zhou C, et al. Gut microbiome remodeling induces depressive-like behaviors through a pathway mediated by the host's metabolism. Mol Psychiatry. 2016;21(6):786-796.

17. Zheng P, Chen JJ, Zhou CJ, et al. Identification of sex-specific urinary biomarkers for major depressive disorder by combined application of NMR- and GC-MS-based metabonomics. Transl Psychiatry. 2016; 6:e955.

18. Yurkovetskiy L, Burrows M, Khan AA, et al. Gender bias in autoimmunity is influenced by microbiota. Immunity. 2013;39:400-412. 
19. Labonté B, Engmann O, Purushothaman I, et al. Sex-specific transcriptional signatures in human depression. Nat Med. 2017;23:1102-1111.

20. Williams JB. A structured interview guide for the Hamilton Depression Rating Scale. Arch Gen Psychiatry. 1988;45:742-747.

21. Tamaki H, Wright CL, Li X, et al. Analysis of $16 \mathrm{~S}$ rRNA amplicon sequencing options on the Roche/454 next-generation titanium sequencing platform. PLoS One. 2011;6:e25263.

22. Yang S, Liebner S, Alawi M, Ebenhöh O, Wagner D. Taxonomic database and cut-off value for processing morA gene 454 pyrosequencing data by Mothur. J Microbiol Methods. 2014;103:3-5.

23. Cole JR, Wang Q, Fish JA, et al. Ribosomal Database Project: data and tools for high throughput rRNA analysis. Nucleic Acids Res. 2013;42: D633-D642.

24. Keylock C. Simpson diversity and the Shannon-Wiener index as special cases of a generalized entropy. Oikos. 2005;109:203-207.

25. Lozupone C, Knight R. UniFrac: a new phylogenetic method for comparing microbial communities. Appl Environ Microbiol. 2005;71: 8228-8235.

26. Segata N, Izard J, Waldron L, et al. Metagenomic biomarker discovery and explanation. Genome Biol. 2011;12:R60.

27. Parker G, Fletcher K, Paterson A, Anderson J, Hong M. Gender differences in depression severity and symptoms across depressive sub-types. J Affect Disord. 2014;167:351-357.

28. Cáceda R, Moskovciak T, Prendes-Alvarez S, et al. Gender-specific effects of depression and suicidal ideation in prosocial behaviors. PLoS One. 2014;9:e108733.

29. Mackay CE, Roddick E, Barrick TR, et al. Sex dependence of brain size and shape in bipolar disorder: an exploratory study. Bipolar Disord. 2010; 12:306-311.

30. Chen JJ, Huang H, Zhao LB, et al. Sex-specific urinary biomarkers for diagnosing bipolar disorder. PLoS One. 2014;9(12):e115221.

31. Butnoriene J, Bunevicius A, Norkus A, Bunevicius R. Depression but not anxiety is associated with metabolic syndrome in primary care based community sample. Psychoneuroendocrinology. 2014;40: 269-276.

32. Bailey MT, Dowd SE, Galley JD, Hufnagle AR, Allen RG, Lyte M. Exposure to a social stressor alters the structure of the intestinal microbiota: implications for stressor-induced immunomodulation. Brain Behav Immun. 2011;25:397-407.

33. Ley RE, Turnbaugh PJ, Klein S, Gordon JI. Microbial ecology: human gut microbes associated with obesity. Nature. 2006;444:1022-1023.
34. Stunkard AJ, Faith MS, Allison KC. Depression and obesity. Biol Psychiatry. 2003;54:330-337.

35. Troseid M, Nestvold TK, Rudi K, Thoresen H, Nielsen EW, Lappegård KT. Plasma lipopolysaccharide is closely associated with glycemic control and abdominal obesity: evidence from bariatric surgery. Diabetes Care. 2013;36:3627-3632.

36. Schnorr SL, Bachner HA. Integrative therapies in anxiety treatment with special emphasis on the gut microbiome. Yale J Biol Med. 2016;89: 397-422.

37. McMillin M, Frampton G, Quinn M, et al. Suppression of the HPA axis during cholestasis can be attributed to hypothalamic bile acid signaling. Mol Endocrinol. 2015;29:1720-1730.

38. Rogers GB, Keating DJ, Young RL, Wong ML, Licinio J, Wesselingh S. From gut dysbiosis to altered brain function and mental illness: mechanisms and pathways. Mol Psychiatry. 2016;21:738-748.

39. Ben-Ari Y. Neuropaediatric and neuroarchaeology: understanding development to correct brain disorders. Acta Paediatr. 2013;102:331-334.

40. Erny D, de Angelis AL, Jaitin D, et al. Host microbiota constantly control maturation and function of microglia in the CNS. Nat Neurosci. 2015; 18:965-977.

41. Sobko T, Huang L, Midtvedt T, et al. Generation of NO by probiotic bacteria in the gastrointestinal tract. Free Radic Biol Med. 2006; 41:985-991.

42. Kamiya T, Wang L, Forsythe P, et al. Inhibitory effects of Lactobacillus reuteri on visceral pain induced by colorectal distension in SpragueDawley rats. Gut. 2006;55:191-196.

43. Rousseaux C, Thuru X, Gelot A, et al. Lactobacillus acidophilus modulates intestinal pain and induces opioid and cannabinoid receptors. Nat Med. 2007;13:35-37.

44. Human Microbiome Project Consortium. Structure, function and diversity of the healthy human microbiome. Nature. 2012;486:207-214.

45. Baker JM, Al-Nakkash L, Herbst-Kralovetz MM. Estrogen-gut microbiome axis: physiological and clinical implications. Maturitas. 2017; 103:45-53.

46. Cubała WJ, Landowski J. C-reactive protein and cortisol in drug-naïve patients with short-illness-duration first episode major depressive disorder: possible role of cortisol immunomodulatory action at early stage of the disease. J Affect Disord. 2014;152-154:534-537.
Neuropsychiatric Disease and Treatment

\section{Publish your work in this journal}

Neuropsychiatric Disease and Treatment is an international, peerreviewed journal of clinical therapeutics and pharmacology focusing on concise rapid reporting of clinical or pre-clinical studies on a range of neuropsychiatric and neurological disorders. This journal is indexed on PubMed Central, the 'PsycINFO' database and CAS,

\section{Dovepress}

and is the official journal of The International Neuropsychiatric Association (INA). The manuscript management system is completely online and includes a very quick and fair peer-review system, which is all easy to use. Visit http://www.dovepress.com/testimonials.php to read real quotes from published authors. 